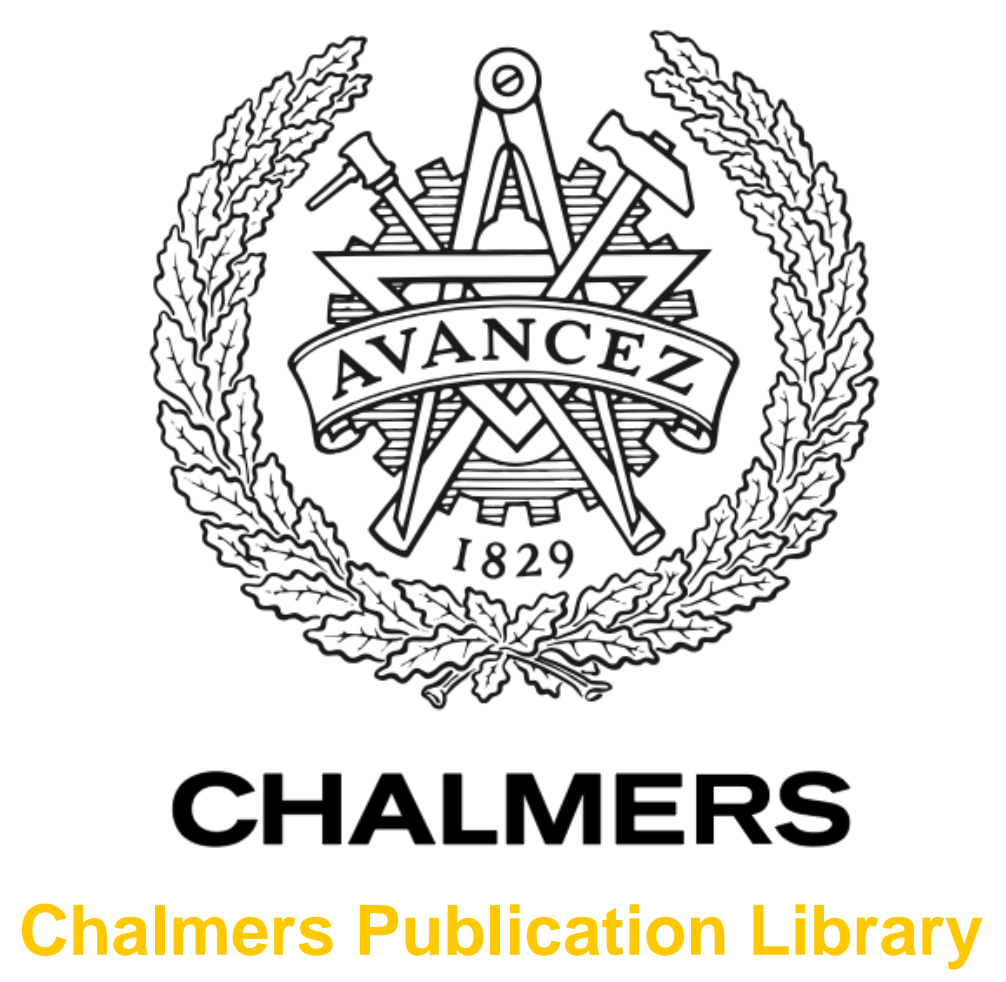

\title{
Fiber nonlinearity mitigation in WDM systems: Strategies and achievable rates [Invited]
}

This document has been downloaded from Chalmers Publication Library (CPL). It is the author's version of a work that was accepted for publication in:

\section{European Conference on Optical Communication (ECOC)}

Citation for the published paper:

Secondini, M. ; Agrell, E. ; Forestieri, E. et al. (2017) "Fiber nonlinearity mitigation in WDM systems: Strategies and achievable rates [Invited]". European Conference on Optical

Communication (ECOC)

Downloaded from: http://publications.lib.chalmers.se/publication/254748

Notice: Changes introduced as a result of publishing processes such as copy-editing and formatting may not be reflected in this document. For a definitive version of this work, please refer to the published source. Please note that access to the published version might require a subscription. 


\title{
Fiber Nonlinearity Mitigation in WDM Systems: Strategies and Achievable Rates
}

\author{
Marco Secondini ${ }^{(1)}$, Erik Agrell ${ }^{(2)}$, Enrico Forestieri ${ }^{(1)}$, Domenico Marsella ${ }^{(3)}$ \\ (1) TeCIP Institute, Scuola Superiore Sant'Anna, Pisa, Italy, name.surname@santannapisa.it \\ (2) Department of Electrical Engineering, Chalmers University of Technology, Gothenburg, Sweden. \\ (3) Nokia, Vimercate, Italy. With (1) at the time of this research.
}

\begin{abstract}
After reviewing models and mitigation strategies for interchannel nonlinearity, the synergic effect of symbol-rate optimization and phase-noise compensation is investigated. This effect can be practically exploited for nonlinearity mitigation and for the computation of capacity lower bounds.
\end{abstract}

\section{Introduction}

Channel models are of paramount importance in the design of optimum detection strategies and in the determination of capacity limits. In optical fiber communications, channel modeling and capacity evaluation are still open problems ${ }^{1}$, and a few different approximated channel models coexist $^{2-6}$ (and references therein), often with a conflicting view about the nature of nonlinear effects. A quite common trend is that of representing complicated effects, such as fiber nonlinearity, as an additional source of additive white Gaussian noise $(\text { AWGN })^{2,6}$. This approach, besides simplifying the analysis, usually provides a satisfactory accuracy when used to predict the performance of conventional systems. The main reason of this accuracy becomes apparent when replacing the ambiguous concept of "conventional" with the more explicit one of "designed for the AWGN channel". However, extending this approach to capacity analysis, which entails considering the best possible combination of modulation, coding, and detection, is not likely to give the desired results. In fact, it only provides a capacity lower bound-the socalled nonlinear Shannon limit-which is actually achievable by conventional systems, but possibly very loose ${ }^{7,8}$. No matter how accurate AWGN-like models might seem, if we aim for tighter capacity lower bounds or better modulation and detection strategies, we have to drop the AWGN assumption and look for any non-AWGN channel characteristics that can be exploited to improve system performance. Such an attempt is made in this work.

By resorting to the frequency-resolved logarithmic perturbation (FRLP) model ${ }^{4,5}$, which describes interchannel nonlinearity in optical fibers through a doubly dispersive fading channel model, we devise a simple nonlinearity mitigation strategy based on the synergic effect of phase noise (PN) compensation and symbol rate optimization in subcarrier multiplexing (SCM). Contrary to what is predicted by AWGN-like models, this synergy persists also for higher-order modulation formats and Gaussian symbols ${ }^{9-11}$, which allows us to derive tighter capacity lower bounds compared to

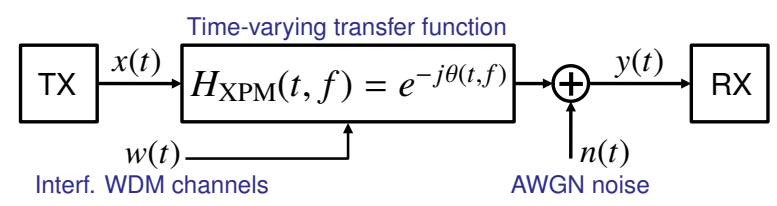

Fig. 1: Continuous-time FRLP model.

the classical nonlinear Shannon limit. The dependence of the achievable gains on the amplification profile is also studied. Finally, possible improvements and alternative approaches worth to be investigated are discussed.

\section{Modeling and mitigation of fiber nonlinearity}

A schematic representation of the (continuoustime) FRLP model for WDM systems is shown in Fig. 1: $x(t)$ and $y(t)$ represent the channel of interest (COI) at the input and output of the fiber link, respectively; the time-varying transfer function $H_{\mathrm{XPM}}(f, t)=e^{-j \theta(f, t)}$ models the effect of crossphase modulation (XPM) through the XPM term $\theta(f, t)$, which depends on the other WDM channel(s) $w(t)$ at the input of the link; and $n(t)$ is the AWGN due to optical amplifiers. Some general expressions for $\theta(f, t)$ and its statistics are provided in $^{4,5}$. For simplicity, the model assumes propagation in a single polarization, such that all involved quantities are scalar. Other non-explicitly modeled propagation effects are assumed to have been either compensated for (e.g., chromatic dispersion and deterministic intrachannel nonlinear effects) or included in the AWGN term by increasing its power spectral density (e.g., four-wave mixing). From the $\mathrm{COI}$ viewpoint, the nonlinear time-invariant optical fiber channel is seen as a linear time-varying one. This seeming paradox is explained by the fact that channel nonlinearity is accounted for by the quadratic dependence of $\theta(f, t)$ on $w(t)^{5}$. However, assuming that $w(t)$ is unknown to both the transmitter and the receiver, such nonlinearity remains hidden, and the effect of the XPM term is simply perceived as linear intersymbol interference (ISI) with unknown (random) coefficients. On the other hand, since $w(t)$ depends on time, the ISI coefficients are time-varying. The model in Fig. 1 is substantially that of a doubly dispersive 


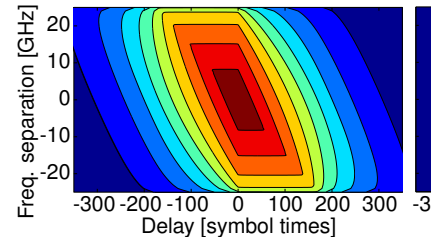

(a) (b)

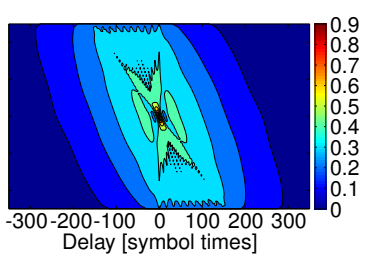

Fig. 2: XPM coherence: (a) IDA link; (b) 10x100km LA link.

fading channel, often used in wireless communications, whose key features are the coherence time and bandwidth over which the channel remains strongly correlated ${ }^{12}$. This analogy may help to better understand the channel characteristics and behavior, as well as to devise improved transmission and detection strategies.

The coherence properties of the channel are shown in Fig. 2, considering a Nyquist-WDM system with $50 \mathrm{GHz}$ channel spacing and bandwidth, Gaussian symbols (as we are interested in highorder modulations and capacity bounds) and a $1000 \mathrm{~km}$ link of standard single-mode fiber with either ideal distributed amplification (IDA) (a) or 10x100 km lumped amplification (LA) (b). The contour plots show the correlation between the values $\theta(0, t)$ and $\theta(\Delta f, t+\tau)$ of the XPM term at two different times and frequencies inside the COI bandwidth, as a function of the delay $\tau$ and frequency separation $\Delta f$. Only the XPM term generated by the couple of closest interfering channels (i.e., those located at $f= \pm 50 \mathrm{GHz}$ ) is considered. The coherence is quite substantial in the IDA link, but significantly reduced in the LA link.

The long coherence time of the XPM term in IDA links was analytically predicted in ${ }^{4}$, suggesting that it could be exploited for a partial mitigation of interchannel nonlinearity and a subsequent increase of the achievable information rate (AIR). For instance, an improved capacity lower bound was obtained in ${ }^{13}$ by PN compensation, while a larger AIR gain was obtained in ${ }^{14}$ by linear equalization (addressing the ISI generated by frequency selectivity) combined with recursive-least-squares adaptation (accounting for time selectivity).

In wireless communications, frequency selectivity is typically addressed by employing multicarrier modulation. By using many low-rate orthogonal carriers, ISI is avoided and the channel response is accounted for by a single-tap equalizer. On the other hand, time selectivity breaks the orthogonality between subcarriers, causing interchannel interference $(\mathrm{ICl})$. In this case, a good trade off between ISI and ICI can be found by optimizing the number of carriers. In Nyquist-WDM, each WDM channel can be further decomposed into many subcarriers, all electronically generated by the same transmitter and jointly processed by the same receiver. A simple approximated discretetime model for the COI of a WDM system employing SCM is shown in Fig. 3. It is derived from the continuous-time model in Fig. 1 by assuming

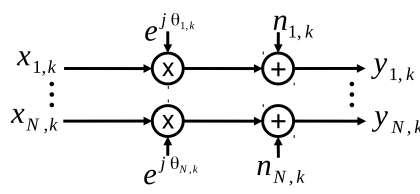

Fig. 3: Discrete-time model of the SCM system (only the COI).

that the XPM term is approximately constant over each subcarrier bandwidth (thus neglecting ISI) and symbol-time (thus neglecting $\mathrm{ICl}$ ), thus manifesting itself as pure PN. On the generic subcarrier $i$, the output sample $y_{i, k}$ at discrete time $k$ is a replica of the input symbol $x_{i, k}$, corrupted by PN $\theta_{i, k}$ and AWGN $n_{i, k}$. PN processes represent the discretized version of the XPM term $\theta(f, t)$. Based on the statistics of $\theta(f, t)^{5}$, they are assumed to be Gaussian, independent (correlation between subcarriers is present, as shown in Fig. 2, but neglected), and follow a (wrapped) first-order autoregressive model $A R(1)$ with subcarrier-dependent parameters. AWGN processes account for the accumulated noise from optical amplifiers, as well as for the other effects induced by fiber nonlinearity and not explicitly included in the model (e.g, fourwave mixing and the aforementioned ISI and $\mathrm{ICI}$ between subcarriers). Thus, they are independent and have different variances. Resorting to this model, it is possible to improve the detection strategy and optimize the number of SCM subcarriers to minimize the ISI and $\mathrm{ICl}$ caused by $\mathrm{XPM}^{9}$.

\section{Achievable information rate}

In this section, we compute the AIR under constrained modulation and mismatched decoding, based on the auxiliary-channel lower bound (to mutual information and capacity) defined in ${ }^{15}$. We consider the same Nyquist-WDM system considered in the previous section, with five channels. We select the central channel as the $\mathrm{COI}$ and, employing SCM, divide it into $N$ subcarriers with rectangular spectrum. The same input distribution and launch power is assumed for all the WDM channels (behavioral model (c) in ${ }^{7}$ ). At the receiver, after single-channel ideal backpropagation, the $N$ subcarriers of the COI are selected by a bank of filters and independently processed. The AIR (averaged over all the subcarriers) is computed for Gaussian input symbols and a detector optimized for the auxiliary channel in Fig. 3 (PN detector hereinafter). Being computational complexity not of primary interest in this analysis, an $L$-state auxiliary channel is used to account for an $L$-level discretized version of PN ( $L=256$ was enough to avoid any AIR loss). All the parameters characterizing the AWGN and PN processes in Fig. 3 are estimated by maximum likelihood estimation during a preliminary training phase.

Fig. 4(a) shows the AIR as a function of the launch power for different numbers of subcarriers $N$. As a comparison, we also report the AIR obtained with an AWGN detector (i.e., optimized for the AWGN channel). Note that, for Gaussian in- 


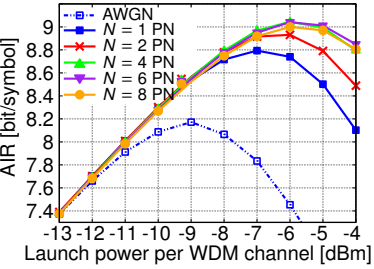

(a)

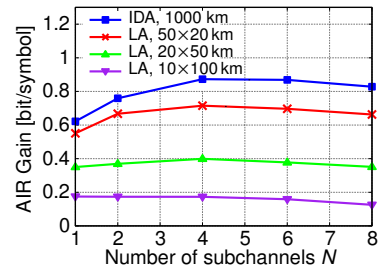

(b)
Fig. 4: (a) AIR for the IDA link; (b) AIR gain for various span lengths. AIR values equal spectral efficiencies in bit/s/Hz.

put symbols, changing $N$ does not alter the input distribution. As a consequence, also the amount of generated nonlinear interference, the AIR with the AWGN detector, and the (unknown) mutual information over the channel remain unchanged. However, when considering a PN detector, the mismatch between channel and detector depends on $N$. In fact, ISI and ICI, unaccounted for by the model, respectively decreases and increases by increasing $N$. An optimum value of $N$ exists which, minimizing the overall interference, minimizes the mismatch and maximizes the AIR. Thanks to the large coherence time and bandwidth of IDA links (see Fig. 2), a significant AIR gain (the difference between the AIR values obtained by the PN and AWGN detectors at their respective optimal launch power) of $\sim 0.9 \mathrm{bit} / \mathrm{symbol}$ is achieved for $N=4$, in fact obtaining the tightest known capacity lower bound for the considered channel. Fig. 4(b) shows the AIR gain as a function of $N$ for IDA and LA links with different span lengths. When increasing the span length, the coherence of the XPM term decreases and the AIR gains provided by PN detection and symbol rate optimization reduces.

\section{Discussion and conclusions}

We have discussed the importance of channel models in nonlinearity mitigation, and the need to drop the AWGN assumption to devise improved detection strategies. A step toward this direction is done by resorting to the FRLP model, which suggests considering interchannel nonlinearity as linear doubly dispersive (in time and in frequency) fading. This analogy helps to understand the characteristics of the channel in terms of its coherence time and bandwidth, and to devise improved transmission schemes borrowed from wireless communications. On this basis, we have derived a simple discrete-time model for SCM systems, which has been used to devise a proper detector, optimize the number of subcarries, and maximize the AIR with Gaussian symbols. Compared to AWGN detection, the AIR gain is maximum for IDA links, and decreases with the span length for LA links. Extension of the results to dual-polarization systems is currently under investigation.

The model also suggests that further optimizations and gains are possible. For instance, accounting for phase correlation among subcarriers could provide some additional AIR gain. Moreover, signaling over short-time Fourier basis functions (generated, via time and frequency shifts, from a prototype pulse with good time and frequency localization properties), possibly combined with $\mathrm{ISI}$ and $\mathrm{ICI}$ cancellation strategies, might be less prone to ISI and ICI compared to standard SCM ${ }^{12}$. Finally, unlike conventional fading, the channel response derived from the FRLP model does not depend on environmental conditions that are out of the user control (e.g., multipath and shadowing effects) but rather on the actual signals transmitted by the other users (channels, according to WDM terminology). Even excluding unpractical joint modulation or detection, this peculiarity gives the possibility of controlling the channel response by adopting a proper combination of coding and modulation on each channel. In this context, an interesting but still unexplored possibility is that of using constellation shaping to increase channel coherence, rather than decrease nonlinear interference. This approach might have a better synergy with the ones discussed in this work and provide additional AIR gains.

\section{References}

[1] E. Agrell et al., "Roadmap of optical communications," J. Optics, Vol. 18, no. 6, p. 063002 (2016).

[2] P. Poggiolini, "The GN model of non-linear propagation in uncompensated coherent optical systems," J. Lightwave Technol., Vol. 30, no. 24, p. 3857 (2012).

[3] A. Mecozzi et al., "Nonlinear Shannon limit in pseudolinear coherent systems," J. Lightwave Technol., Vol. 30, no. 12, p. 2011 (2012).

[4] M. Secondini et al., "Analytical fiber-optic channel model in the presence of cross-phase modulation," IEEE Photon. Technol. Lett., Vol. 24, no. 22, p. 2016 (2012).

[5] M. Secondini et al., "Achievable information rate in nonlinear WDM fiber-optic systems with arbitrary modulation formats and dispersion maps," J. Lightwave Technol., Vol. 31, no. 23, p. 3839 (2013).

[6] A. Carena et al., "EGN model of non-linear fiber propagation," Optics Express, Vol. 22, no. 13, p. 16335 (2014).

[7] E. Agrell et al., "Influence of behavioral models on multiuser channel capacity," J. Lightwave Technol., Vol. 33, no. 17 , p. 3507 (2015)

[8] M. Secondini et al., "Scope and limitations of the nonlinear Shannon limit," J. Lightwave Technol., Vol. 35, no. 4, p. 893 (2017).

[9] D. Marsella et al., "A simple strategy for mitigating XPM in nonlinear WDM optical systems," Proc. Opt. Fiber Commun. Conf. (OFC), Paper Th4D.3, (2015).

[10] R. Dar et al., "Nonlinear interference mitigation: methods and potential gain," J. Lightwave Technol., Vol. 35, no. 4, p. 903 (2017).

[11] F. Guiomar et al., "Effectiveness of symbol-rate optimization with PM-16QAM subcarriers in WDM transmission," Proc. Opt. Fiber Commun. Conf. (OFC), Optical Society of America (2017) W3J.3.

[12] K. Liu et al., "Orthogonal time-frequency signaling over doubly dispersive channels," IEEE Trans. Inform. Theory, Vol. 50, no. 11, p. 2583 (2004).

[13] R. Dar et al., "New bounds on the capacity of the nonlinear fiber-optic channel," Opt. Lett., Vol. 39, no. 2, p. 398 (2014).

[14] M. Secondini et al., "On XPM mitigation in WDM fiberoptic systems," IEEE Photon. Technol. Lett., Vol. 26, no. 22, p. 2252 (2014).

[15] D. M. Arnold et al., "Simulation-based computation of information rates for channels with memory," IEEE Trans. Inform. Theory, Vol. 52, no. 8, p. 3498 (2006). 